\title{
Functional Polymorphisms of CTLA4 Associated with Aggressive Periodontitis in the Chinese Han Population
}

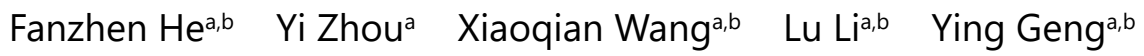 \\ Zhenting Wang ${ }^{\mathrm{a}}$ Yuzhen Wang ${ }^{\mathrm{a}}$ Yan $\mathrm{Xu}^{\mathrm{a}, \mathrm{b}}$ \\ ajiangsu Key Laboratory of Oral Disease, Nanjing Medical University, Nanjing, ${ }^{\text {bDepartment of }}$ \\ Periodontics, The Affiliated Stomatological Hospital of Nanjing Medical University, Nanjing, China
}

\section{Key Words}

Polymorphism • miR-105 • 3'-UTR • Periodontitis •Immune

\begin{abstract}
Background/Aims: CTLA4 has been identified functioning as a protein receptor which functions as an immune checkpoint, downregulating the immune system. Susceptibility to aggressive periodontitis (AgP) is influenced by gene polymorphisms related to the immune response. In this study, we focused on SNPs in the 3'-UTR of CTLA4 among Chinese AgP patients, and investigated any further relationships between the SNPs and miRNAs. Methods: This case-control study included $120 \mathrm{AgP}$ patients and 150 healthy controls. Genotyping was used to detect allele distribution. Cell transfection and the dual luciferase reporter assay were performed to investigate the potential functions of SNPs located in the 3'UTR of CTLA4. Results: The data show that patients with a history of smoking were more susceptible compared to controls, exhibiting deeper probing depth, greater attachment loss and more sites of bleeding on probing. The results of genotyping analysis revealed that individuals with the GA and AA genotypes, and with the A carrier had a decreased risk $(P=0.015, P=0.03)$. Furthermore, patients with the $G$ allele might be regulated by miR-105, which caused a down-regulation of CTLA4. The carriers of the GG genotype exhibited the worst results of attachment loss and bleeding on probing. Conclusion: These findings show that rs56102377 in the 3'-UTR of CTLA4 may act as a protective factor by disrupting the regulatory role of miR-105 in CTLA4 expression. Thus, our study highlighted a potential role of these polymorphisms as genetic susceptibility biomarkers of periodontitis in Chinese Han populations.
\end{abstract}

\begin{tabular}{ll}
\hline Yan Xu & Department of Periodontics, The Affiliated Stomatological Hospital of Nanjing Medical University, \\
& 136 Han Zhong Road, Nanjing, Jiangsu, 210029 (China) \\
E-Mail yanxu@njmu.edu.cn
\end{tabular}




\section{Introduction}

Characterized by a destructive inflammatory process, periodontal disease is a multifactorial infectious inflammatory condition which affects the tooth-supporting tissues, resulting in periodontal pocket formation, alveolar bone resorption and eventually tooth loss $[1,2]$. It has been demonstrated that environmental factors such as hormones, drugs or diet modify preexisting periodontal conditions [3]. Among these factors, smoking is considered one of the main risk factors for periodontitis. Smoking affects the oral environment, gingival vasculature, and inflammatory and immunological responses, as well as the healing potential of periodontal connective tissues [4,5].

Recent studies have demonstrated that inflammatory cytokines, including IL-1, IL6, TNF-alpha and IFN-gamma, are risk factors for aggressive periodontitis [6-8]. The relationship between host immune response and periodontal pathogens plays a critical role in periodontal tissue breakdown $[9,10]$. Protective and destructive immune responses play a great role in the pathogenesis of periodontitis and attachment loss. Thus, relationships between genes regulating the immune responses and periodontitis seem to be intuitively reasonable [11]. Cytotoxic T-lymphocyte antigen 4 (CTLA4), encoded by the gene CTLA4, belongs to the immunoglobulin supergene family [12]. In recent years, the relationship between CTLA4 polymorphisms and some diseases such as Graves' disease and hepatocellular carcinoma has attracted extensive attention $[13,14]$. Previous studies indicated that CTLA4 polymorphisms were closely related to various autoimmune diseases such as idiopathic dilated cardiomyopathy and lupus erythematous as well as thyroid diseases $[15,16]$. In addition, T (-) in CD28 + $17(\mathrm{~T} / \mathrm{C})$ and A (+) in CTLA4 +49 (A/G) genotypes are associated with susceptibility to aggressive periodontal disease and CTLA4 gene variants might be associated with susceptibility to a specific form of periodontitis [17]. However, most of the SNPs reported in CTLA4 are located in the coding region; the association of any SNPs located in the non-coding region, especially the 3' untranslated region (UTR), has been poorly investigated. The 3-UTR frequently interacts with microRNAs (miRNAs; non-coding RNA molecules of 19-25 nucleotides) $[18,19]$. The miRNAs negatively regulate expression of their target genes at the posttranscriptional level through binding to 3' UTRs of their targets' messenger RNAs (mRNAs) [20, 21].

Based on the investigations mentioned above, we hypothesized that SNPs in the 3'UTR of CTLA4 may play a role in regulating CTLA4 expression, which may be associated with the pathogenesis of periodontitis. We identified all the SNPs which could be regulated by miRNAs as candidate SNPs by using bioinformatics software (http://www.bioguo.org/miRNASNP/), and the allele distribution was then further investigated in this case-control study.

\section{Materials and Methods}

\section{Study subjects}

This hospital-based case-control study consists of 120 patients newly diagnosed as AgP and 150 healthy controls. All the subjects were genetically unrelated Han Chinese, recruited from the department of Periodontics, Affiliated Stomatological Hospital of Nanjing Medical University between January 2012 and February 2016. The periodontal examination included determination of probing depth (PD), clinical attachment loss (CAL), bleeding on probing (BOP). The subjects who had directly smoked more than 10 cigarettes daily for 5 years were considered as smokers. Measurements of PD and CAL were recorded to the nearest $1 \mathrm{~mm}$, with measures between $1 \mathrm{~mm}$ probe markings rounded down to the nearest length. Measurements were performed at 6 sites per tooth (mesiobuccal, mid-buccal, distobuccal, mesiolingual, mid-lingual, and distolingual). None of patients had a history of current manifestation of systemic diseases. Patients with severe medical disorders (diabetes mellitus, immunological disorders, hepatitis, 


\section{Cellular Physiology Cell Physiol Biochem 2018;50:1178-1185

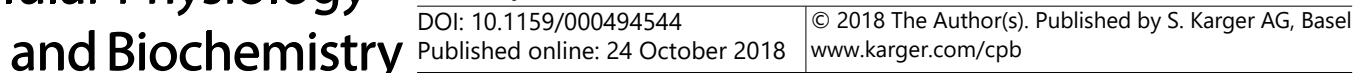 \\ He et al.: SNP in CTLA4 with AGP}

HIV infection, and cardiovascular involvement), use of orthodontic appliances, need for premedication for dental treatment, chronic usage of anti-inflammatory drugs, present acute necrotizing ulcerative gingivitis, and pregnant or lactating woman were excluded from the study. The patients were classified according to World Health Organization classification. This study was approved by Ethics Committee of Nanjing Medical University, every patient had written informed consent.

\section{Genotype}

The genomic DNA obtained from peripheral whole blood of every validation subject was extracted by using QIAamp DNA blood mini kits (QIAGEN, CA, USA) according to the manufacturer's instructions. Genotyping was performed by the TaqMan SNP Genotyping Assay, the PCR reactions were carried out in a total volume of $5 \mu \mathrm{L}$ containing TaqMan Universal Master Mix, 80X SNP Genotyping Assay Mix, Dnase-free water and $10 \mathrm{ng}$ genomic DNA. The PCR conditions were $2 \mathrm{~min}$ at $50^{\circ} \mathrm{C}, 10 \mathrm{~min}$ at $95^{\circ} \mathrm{C}$, followed by 40 cycles at $95^{\circ} \mathrm{C}$ for $15 \mathrm{sec}$ and $60^{\circ} \mathrm{C}$ for $1 \mathrm{~min}$, by using the 384-well ABI 7900HT Real Time PCR System (Thermo, CA, USA).

\section{Real time PCR assay}

Real time polymerase chain reaction (RT-PCR) was performed to determine the expression level of miR-105. The probe for detecting the expression of miR-105 was ordered from Thermo Fisher Scientific with the Cat. \# A25576. The amplification conditions were $95^{\circ} \mathrm{C}$ for 10 minutes, followed by 40 cycles of $95^{\circ} \mathrm{C}$ for 30 seconds, $55^{\circ} \mathrm{C}$ for 40 seconds, and $72^{\circ} \mathrm{C}$ for 30 seconds, and finally $4^{\circ} \mathrm{C}$ for 30 minutes for cooling with the same PCR system mentioned above. The primers for miR-105 were: Forward $5^{\prime}$-GCCCTCG AGATA CC AT ATCTATCCCCTTTTTCA-3'; Reverse $5^{\prime}$-GCCGAATTC CAACCATGA AGAT A CGAATTGATG-3. Primers for GAPDH were: Forward: 5'-GACTCATGACCACAGTCCATGC-3'; Reverse: 3'-A GAGG CAGGGATGATGTTCTG-5'.

\section{Cell lines and cell culture}

Periodontal ligament cell line (PDLC) was purchased from the Science (Science, CA, USA) with the Catalog \#2630. Cells were cultured in Dulbecco Modified Eagle Medium (Gibco, NY, USA) supplemented with $10 \%$ fetal bovine serum (Invitrogen, CA, USA) and grown in humidified $5 \% \mathrm{CO}_{2}$ at $37^{\circ} \mathrm{C}$. The miR105 mimics and normal control were obtained from Genepharma (Shanghai, China). The transfection was conducted by using Lipofectamine 2000 (Invitrogen, CA, USA).

\section{Dual-luciferase reporter gene assay}

The full length of gene CTLA4 containing either $\mathrm{G}$ or A allele was amplified. The PCR productions were cloned into the pGL3-luciferase-based plasmid (Promega, CA, USA). The amplified fragments were verified by DNA sequencing. For reporter assay, cells were plated onto 24-well plates and transfected with $100 \mathrm{ng}$ of wild type pGL3-CTLA4, mutant type pGL3-CTLA4 and miR-105 mimics, respectively by using Lipofectamine 2000 (Invitrogen, CA, USA). A Renilla luciferase vector pRL-SV40 (5 ng) was also co-transfected to normalize the differences in transfection efficiency. Transfection was performed three times in triplicate.

\section{Statistical analysis}

For evaluating the association between rs56102377 genotypes and the risk of AgP, the odds ratios (ORs) and their 95\% confidence intervals (CIs) were calculated by using univariates and multivariate logistic regression analysis. Stratification analysis was performed according to the clinical characteristic and risk classification to determine the genotype distribution as well as their associations with the risk of AgP. All statistical tests were two-sided and $\mathrm{P}<0.05$ was considered statistically significant. Statistical analysis was performed with Stata10.0 and SAS9.1 software. The graphs were generated by GraphPad Prism 6.0. 


\section{Results}

\section{Study population}

The clinical characteristics of all the cases and controls are presented in Table 1. As shown, the study population included $120 \mathrm{AgP}$ patients (65 males, 55 females; age range 19-38 years) and 150 healthy controls (79 males, 71 females; age range 17-50 years). The gender distribution was reasonably well balanced in AgP patients and controls $(P=$ 0.806). The patient population exposed to smoking was indicated to be more susceptible in comparison to controls. The AgP patients exhibited deeper probing depth, more attachment loss and more sites of bleeding on probing.

\section{Correlation of rs56102377 with susceptibility to AgP}

CTLA4 has been identified as an important gene in the pathogenesis of AgP, and researchers have found that the T- in CD28 +17 (T/C) and the A+ in CTLA4 +49 (A/G) genotypes are associated with susceptibility to aggressive periodontal disease [17]. However, the SNPs reported were all located in the coding region of CTLA4, while there have been few investigations concerning the non-coding region of CTLA4. First, we screened the potential SNPs located in the 3'UTR region of CTLA4 and identified possible candidates binding any miRNA which has been shown to have a possible association with the polymorphism in a public database. In our results, the only SNP located in the 3'UTR region of CTLA4 was rs56102377, which may be associated with interrupting miR-105 binding to CTLA4.

Table 1. Frequency distributions of selected variables in AgP patients and healthy controls. a Student $t$ test b Two-sided chi-square test

\begin{tabular}{lccc}
\hline Variables & $\operatorname{AgP}(\mathrm{n}=120)$ & Control $(\mathrm{n}=150)$ & $\mathrm{P}$ \\
\hline Age (years) & $27 \pm 10$ & $29 \pm 12$ & $0.132^{\mathrm{a}}$ \\
Gender & 65 & & $0.806^{\mathrm{b}}$ \\
Male & 55 & 79 & \\
Female & & & $<0.0001^{\mathrm{b}}$ \\
Smoking exposure & 82 & 133 & \\
Negative & 38 & 17 & \\
Positive & $6.38 \pm 1.689$ & $1.66 \pm 1.338$ & $<0.0001^{\mathrm{a}}$ \\
Probing depth (mm) & $6.68 \pm 1.332$ & $0.81 \pm 1.102$ & $<0.0001^{\mathrm{a}}$ \\
Attachment loss (mm) & $82.1 \pm 8.53$ & $8.9 \pm 0.56$ & $<0.0001^{\mathrm{a}}$ \\
Bleeding on probing $(\%)$ & & & \\
\hline
\end{tabular}

Table 2. Genotype frequencies of the CTLA4 rs56102377 polymorphism among AgP patients and controls. aThe 0Rs, 95\% CIs and P value were calculated after adjusting for age, gender, parental smoking history, probing depth, attachment loss, bleeding on probing

\begin{tabular}{lcccccc}
\hline Genotype & \multicolumn{2}{c}{ Cases $(\mathrm{n}=120)$} & \multicolumn{2}{c}{ Controls $(\mathrm{n}=150)$} & $\begin{array}{c}\text { OR } \\
(95 \% \mathrm{CI}) \mathrm{a}\end{array}$ & P Value $^{\mathrm{a}}$ \\
& $\mathrm{N}$ & $\%$ & $\mathrm{~N}$ & $\%$ & & \\
\hline rs56102377 & & & & & & \\
& & & & & & \\
GG & 57 & 47.5 & 81 & 54.0 & 1.00 & 0.015 \\
GA & 50 & 41.7 & 65 & 43.3 & $0.62(1.11-1.34)$ & \\
AA & 13 & 10.8 & 4 & 2.7 & $0.77(1.25-1.71)$ & \\
A carrier & 63 & 52.5 & 69 & 46.0 & $0.68(1.19-1.64)$ & 0.03 \\
\hline
\end{tabular}


Table 3. Stratified analysis of rs56102377 genotype with clinicopathological parameters of AgP. *Two-sided chi-square test for either genotype distributions or allele frequencies between cases and controls

\begin{tabular}{lccccccc}
\hline Feather & \multicolumn{9}{c}{ Genotype } & GA vs GG & AA vs GG & $\begin{array}{c}\text { A carrier vs GG } \\
\text { P Value }\end{array}$ \\
& GG & GA & AA & A carrier & P Value & P Value & ( \\
\hline Gender & & & & & & & \\
Male & 32 & 26 & 7 & 33 & 0.668 & 0.881 & 0.680 \\
Female & 25 & 24 & 6 & 30 & & & \\
Attachment loss (mm) & & & & & & & \\
Mild & 17 & 33 & 10 & 43 & 0.0001 & 0.002 & $<0.0001$ \\
Serious & 40 & 17 & 3 & 20 & & & \\
Bleeding on probing (\%) & & & & & & & \\
& 21 & 30 & 9 & 39 & 0.017 & 0.03 & 0.006 \\
Mild & 36 & 20 & 4 & 24 & & & \\
Serious & & & & & & & \\
\hline
\end{tabular}

Fig. 1. Functional SNP causing incapacitation of miR-105 binding. A: Bioinformatics predicted a binding site between miR-105 and CTLA4 and the mutation types were transfected into the pGL3 plasmid as shown. B: Periodontal ligament cells were co-transfected with miR-105 mimics or control, with Renilla luciferase vector pRLSV40 for $48 \mathrm{~h}$. Both firefly and Renilla luciferase activities were measured in the same sample.

A

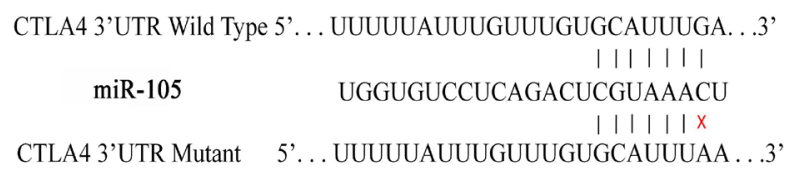

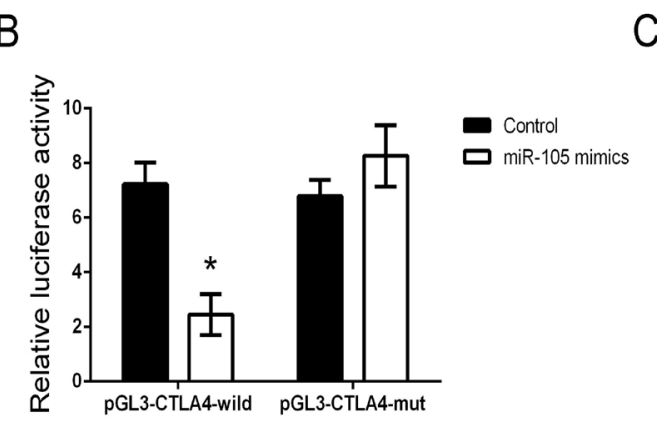

C

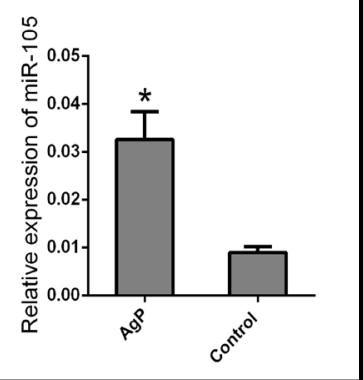

Firefly luciferase signals

were normalized to Renilla luciferase signals. C: Relative expression level of miR-105 in the serum of AgP patients detected by RT-PCR. Data are presented as the mean \pm SEM. * indicates a significant difference $(\mathrm{P}<0.05)$.

Next, we analyzed the candidate genotype frequency in 120 patients and 150 controls. As listed in Table 2, the results of Chi-square statistical analysis showed that the genotype distribution of rs56102377 was in Hardy-Weinberg equilibrium in the healthy control group $(P=0.54)$. Logistic regression analysis results revealed that individuals with the GA and AA genotype were significantly associated with $\mathrm{AgP}$ risk $(\mathrm{OR}=0.62,95 \% \mathrm{CI}=1.11-1.34$ and $\mathrm{OR}$ $=0.77,95 \% \mathrm{CI}=1.25-1.71 ; P=0.015)$, indicating that the GA and AA genotypes presented $\mathrm{a}$ significantly decreased risk of AgP compared to the GG genotype. In addition, the A carrier also indicated a decreased risk $(P=0.03)$ with $\mathrm{OR}=0.68,95 \% \mathrm{CI}=1.19-1.64$. All ORs were adjusted for sex, age, and smoking status, probing depth, attachment loss, and bleeding on probing. 
Stratified analysis of SNP rs56102377 in AgP patients

A stratified analysis was performed between the distributions of patients with different genotypes and the clinical parameters of AgP (Table 3). A significant association was found between the rs56102377 genotype and clinical attachment loss and probing bleeding. Compared to GG homozygotes, the carriers of GA and AA genotypes presented with more attachment loss and probing bleeding, which indicated that SNP rs56102377 located in the 3'UTR of CTLA4 may serve as a protective factor in the pathogenesis of AgP.

\section{Interaction of miR-105 with SNP rs56102377 in CTLA4}

Based on the distributions of SNP rs56102377 in patients and controls, we hypothesized that the expression of CTLA4 might be dysregulated. We assumed that the SNP rs56102377 might affect the expression of CTLA4, as the speculated location of this SNP was in the binding site of miR-105. The detailed binding site of miR-105 in the 3'UTR of CTLA4 is shown in Fig. $1 \mathrm{~A}$, both wild type (with allele $\mathrm{G}$ ) and mutant type (with allele $\mathrm{A}$ ) with full length CTLA4 cloned into the pGL3 plasmid tagged with luciferase. When periodontal ligament cells were co-transfected with miR-105 mimics with either wild-type or mutant type CTLA4 allele, the expression of CTLA4 was decreased in cells treated with wild-type CTLA4 compared to mutant type or the control group (Fig. 1B). The expression of miR-105 in the serum samples revealed that miR-105 was upregulated in AgP (Fig. 1C). Thus, it might be reasonable to assume that the increased level of miR-105 could suppress CTLA4 level by binding to its 3'UTR region, while the SNP located in the binding sites might cause incapacitation, resulting in higher expression of CTLA4.

\section{Discussion}

There is increasing evidence that SNPs located in the binding sites of miRNAs (miRSNPs) may cause a reduction or increase in the translation of the target mRNA by affecting the binding of a miRNA to its target genes, thus altering the susceptibility to disease $[22,23]$. Researchers have found that the rs2910164 polymorphism in the miR-146a precursor sequence may influence the susceptibility of the Chinese population to gastric cancer, while the SNPs rs4143815 and rs4819388 in the 3'UTRs of B7-H1 and B7-H2 genes are significantly related to the occurrence of gastric cancer $[24,25]$. In this study, we found that CTLA4 with SNP rs56102377 resulting in a GA or AA genotype is associated with a significantly lower rate of AgP compared to the GG genotype. We also found that SNP rs56102377 located in the binding sites of increased circulating miR-105 played an important role in the development of AgP by disrupting the inhibitory role of miR-105 on CTLA4 expression.

The 2 q33 chromosomal region bears a cluster of genes, including the CD28, CTLA4, and inducible T-cell costimulator (ICOS), with related functions in T-cell immune regulation. Several SNPs have been identified in the promoter region of the human CTLA4 gene $(+1722 \mathrm{~T} / \mathrm{C},+1661 \mathrm{~A} / \mathrm{G},+318 \mathrm{C} / \mathrm{T})$ and in exon $1(+49 \mathrm{G} / \mathrm{A})$, some of which are related to differences in CTLA4 expression or have been associated with susceptibility to autoimmune and infectious diseases [12]. CTLA4 plays a significant role in regulating peripheral T-cell tolerance and attenuating T-cell response. CTLA4/B7 interactions block T-cell activation and promote T-cell death or apoptosis. CTLA4 suppresses the proliferation of T-cells in response to periodontopathogens. The present finding suggests that the presence of a native CTLA4 molecule on the surface of Tregs in the normal population harboring an A allele would produce appropriate regulatory/inhibitory responses which control or attenuate inflammatory responses against periodontopathogens or antigens while bearing a $\mathrm{G}$ allele in this position would not. 


\begin{tabular}{|c|c|}
\hline Cellular Physiology & Cell Physiol Biochem 2018;50:1178-1185 \\
\hline and Biochemistry & \begin{tabular}{l|l} 
DOl: 10.1159/000494544 & $\begin{array}{l}\text { O } 2018 \text { The Author(s). Published by S. Karger AG, Basel } \\
\text { Published online: } 24 \text { October } 2018 \\
\text { wwww.karger.com/cpp }\end{array}$ \\
\end{tabular} \\
\hline
\end{tabular}

\section{Conclusion}

In summary, we found a functional SNP located in the 3'UTR of CTLA4 which might cause incapacitation binding of miR-105, thus resulting in an increased level of CTLA4 in AgP patients. Further, SNP rs56102377 in CTLA4 may act as a protective factor in the pathogenesis of AgP.

\section{Acknowledgements}

This study was supported by grants from National Natural Science Foundation of China (Grants No. 81470749\&81771074), the project funded by the Priority Academic Program Development of Jiangsu Higher Education Institutions (PAPD, 2014-37), and the Major Program of the Natural Science Foundation of the Jiangsu Higher Education Institutions of China (Grant No.16KJA320001).

\section{Disclosure Statement}

The authors declare no conflicts of interest.

\section{References}

1 Hajishengallis G: Periodontitis: From microbial immune subversion to systemic inflammation. Nat Rev Immunol 2015;15:30-44.

-2 Subramanian S, Emami H, Vucic E, Singh P, Vijayakumar J, Fifer KM, Alon A, Shankar SS, Farkouh M, Rudd JH, Fayad ZA, Van Dyke TE, Tawakol A: High-dose atorvastatin reduces periodontal inflammation: A novel pleiotropic effect of statins. J Am Coll Cardiol 2013;62:2382-2391.

3 Allin N, Cruz-Almeida Y, Velsko I, Vovk A, Hovemcamp N, Harrison P, Huang H, Aukhil I, Wallet SM, Shaddox LM: Inflammatory response influences treatment of localized aggressive periodontitis. J Dent Res 2016;95:635-641.

4 Wu L, Zhou Y, Zhou Z, Liu Y, Bai Y, Xing X, Wang X: Nicotine induces theproduction of IL-1 $\beta$ and IL-8 via the $\alpha 7 \mathrm{nAChR} / \mathrm{NF}-\kappa \mathrm{B}$ pathway in human periodontalligament cells: an in vitro study. Cell Physiol Biochem;2014;34:423-431.

5 Wei XM, Chen YJ, Wu L, Cui LJ, Hu DW, Zeng XT: Tumor necrosis factor-alpha g-308a (rs1800629) polymorphism and aggressive periodontitis susceptibility: A meta-analysis of 16 case-control studies. Sci Rep 2016;6:19099.

6 Barnea TV, Sava A, Gentimir C, Goriuc A, Boisteanu O, Chelaru L, Iancu RI, Avram CA, Acatrinei DD, Bogza EG, Raducanu OC, Cioloca DP, Vasincu D, Costuleanu M: Genetic polymorphisms of tnfa and il-1a and generalized aggressive periodontitis. Rom J Morphol Embryol 2015;56:459-464.

7 Chen YJ, Han Y, Mao M, Tan YQ, Leng WD, Zeng XT: Interleukin-1beta rs1143634 polymorphism and aggressive periodontitis susceptibility: A meta-analysis. Int J Clin Exp Med 2015;8:2308-2316.

8 Schulz S, Immel UD, Just L, Schaller HG, Glaser C, Reichert S: Epigenetic characteristics in inflammatory candidate genes in aggressive periodontitis. Hum Immunol 2016;77:71-75.

-9 Gonzales JR, Groeger S, Johansson A, Meyle J: T helper cells from aggressive periodontitis patients produce higher levels of interleukin- 1 beta and interleukin- 6 in interaction with porphyromonas gingivalis. Clin Oral Investig 2014;18:1835-1843.

10 Shaddox LM, Goncalves PF, Vovk A, Allin N, Huang H, Hou W, Aukhil I, Wallet SM: Lps-induced inflammatory response after therapy of aggressive periodontitis. J Dent Res 2013;92:702-708.

11 Velusamy SK, Ganeshnarayan K, Markowitz K, Schreiner H, Furgang D, Fine DH, Velliyagounder K: Lactoferrin knockout mice demonstrates greater susceptibility to aggregatibacter actinomycetemcomitansinduced periodontal disease. J Periodontol 2013;84:1690-1701. 


\section{Cellular Physiology Cell Physiol Biochem 2018;50:1178-1185 \begin{tabular}{l|l|l} 
DOI: 10.1159/000494544 & $\begin{array}{l}\text { O 2018 The Author(s). Published by S. Karger AG, Basel } \\
\text { www.karger.com/cpb }\end{array}$
\end{tabular}}

He et al.: SNP in CTLA4 with AGP

12 Tivol EA, Borriello F, Schweitzer AN, Lynch WP, Bluestone JA, Sharpe AH: Loss of ctla-4 leads to massive lymphoproliferation and fatal multiorgan tissue destruction, revealing a critical negative regulatory role of ctla-4. Immunity 1995;3:541-547.

13 Walker LS: Ctla-4 and autoimmunity: New twists in the tale. Trends Immunol 2015;36:760-762.

14 Liu Z, Song Z, Sun J, Sun F, Li C, Sun J, Xu L: Association between ctla-4 rs231775 polymorphism and hepatocellular carcinoma susceptibility. Int J Clin Exp Pathol 2015;8:15118-15122.

15 Katkam SK, Kumaraswami K, Rupasree Y, Thishya K, Rajasekhar L, Kutala VK: Association of ctla4 exon-1 polymorphism with the tumor necrosis factor-alpha in the risk of systemic lupus erythematosus among south indians. Hum Immunol 2016;77:158-164.

16 McGonagle D, Aziz A, Dickie LJ, McDermott MF: An integrated classification of pediatric inflammatory diseases, based on the concepts of autoinflammation and the immunological disease continuum. Pediatr Res 2009;65:38R-45R.

17 Houshmand B, Rafiei A, Hajilooi M: Influence of cytotoxic t lymphocyte antigen-4 (ctla-4) gene polymorphisms in periodontitis. Arch Oral Biol 2012;57:1218-1224.

18 Dutta B, Azhir A, Merino LH, Guo Y, Revanur S, Madhamshettiwar PB, Germain RN, Smith JA, Simpson KJ, Martin SE, Beuhler E, Fraser ID: An interactive web-based application for comprehensive analysis of rnaiscreen data. Nat Commun 2016;7:10578.

19 Feinberg MW, Moore KJ: Microrna regulation of atherosclerosis. Circ Res 2016;118:703-720.

-20 Rowley JW, Chappaz S, Corduan A, Chong MM, Campbell R, Khoury A, Manne BK, Wurtzel JG, Michael JV, Goldfinger LE, Mumaw MM, Nieman MT, Kile BT, Provost P, Weyrich AS: Dicer1 mediated mirna processing shapes the mrna profile and function of murine platelets. Blood 2016;127:1743-1751.

21 Liu S, da Cunha AP, Rezende RM, Cialic R, Wei Z, Bry L, Comstock LE, Gandhi R, Weiner HL: The host shapes the gut microbiota via fecal microrna. Cell Host Microbe 2016;19:32-43.

22 Gong J, Tian J, Lou J, Ke J, Li L, Li J, Yang Y, Gong Y, Zhu Y, Zhang Y, Zhong R, Chang J, Miao X: A functional polymorphism in lnc-lamc2-1:1 confers risk of colorectal cancer by affecting mirna binding. Carcinogenesis 2016;37:443-451.

23 Ma XP, Yu G, Chen X, Xiao Q, Shi Z, Zhang LY, Chen H, Zhang P, Ding DL, Huang HX, Saiyin H, Chen TY, Lu PX, Wang NJ, Yu H, Conran C, Sun J, Zheng SL, Xu J, Yu L, Jiang DK: Mir-608 rs4919510 is associated with prognosis of hepatocellular carcinoma. Tumour Biol 2016;37:9931-9942.

24 Bastami M, Ghaderian SM, Omrani MD, Mirfakhraie R, Vakili H, Alipour Parsa S, Nariman-Saleh-Fam Z, Masotti A: Mirna-related polymorphisms in mir-146a and tcf21 are associated with increased susceptibility to coronary artery disease in an iranian population. Genet Test Mol Biomarkers 2016;20:241-248.

25 Wang W, Li F, Mao Y, Zhou H, Sun J, Li R, Liu C, Chen W, Hua D, Zhang X: A mir-570 binding site polymorphism in the b7-h1 gene is associated with the risk of gastric adenocarcinoma. Hum Genet 2013;132:641-648. 